\title{
29 \\ Community-based Rehabilitation and Disability-inclusive Development: On a Winding Path to an Uncertain Destination
}

\section{Pim Kuipers}

Menzies Health Institute Queensland, Griffith University and Metro South Health, Australia, p.kuipers@griffith.edu.au

\section{Louis Paluku Sabuni}

The Leprosy Mission, Kinshasa, Democratic Republic of Congo

\section{Introduction}

The majority of people with disabilities who live in developing countries, predominantly in the global South, do not receive any formal disability or rehabilitation services. In those countries or regions where at least some disability services are provided, the communitybased rehabilitation (CBR) approach, or some form of it, is likely to be the only approach available (Evans et al. 2001).

As a formal 'model', CBR was first promoted by the World Health Organization (WHO) in the mid-1970s to address the shortage of rehabilitation services in the global South. A key dimension of the original CBR approach was the transfer of minimum and essential rehabilitation intervention skills to families and other volunteers in the community (Thomas and Thomas 1999). The intent of the model was to provide some form of rehabilitation or disability support services through the local community, using local resources. The approach drew on the principles of primary health care, accepted international rehabilitation practices of the time, and existing local practices and technologies (Hartley et al. 2009). Early CBR practices were based on a few manuals (Helander et al. 1989; Werner 1999) and emphasized basic, individually focused services, such as therapy, education, basic equipment, vocational 
training and referral to medical services (Thomas 2011). Historically CBR has nearly always been promoted and implemented by international non-government organizations (NGOs) based in the global North, such as Handicap International (Handicap International 2014) and CBM (CBM 2014).

The most widely used definition of CBR reflects its change over time, and notes that CBR is:

a strategy within general community development for rehabilitation, equalization of opportunities, and social inclusion of all people with disabilities ... implemented through the combined efforts of people with disabilities themselves, their families and communities, and the appropriate health, education, vocational, and social services ... (ILO et al. 2004)

This statement, emphasizing not just traditional rehabilitation, but including community development, poverty reduction, equalization of opportunities, and social integration, reflects something of the evolution of CBR. In recent years this evolution has been manifest in the growing shift towards the concepts and terminology of disability-inclusive development (DID) by organizations and collaborations that have traditionally emphasized CBR. The evolution towards DID approaches reflects a move beyond traditional rehabilitation to emphasizing rights and inclusion, seeking to ensure that people with disabilities are stakeholders in development processes. The goal of DID is that "all phases of the development cycle (design, implementation, monitoring and evaluation) include a disability dimension and that persons with disabilities are meaningfully and effectively participating in development processes and policies” (IDDC 2010).

Our goal in this chapter is to examine aspects of the evolving nature of CBR, which has facilitated its emergence as a global service model. We hold that CBR and DID approaches and their evolution over past decades have both positive and negative dimensions. Likewise, we see both potentially constructive as well as possibly unproductive directions in the evolutionary paths ahead. We recognize that the CBR model is far from ideal, but we also 
advocate that with adequate vision, the CBR model can and should evolve further to more adequately respond to the needs of people with disability in the global South. Such vision must largely come from the global South, from people with disability, their families and communities.

We conclude that such a 'downstream' focus is what should characterize the evolution of CBR; however, we observe that much of the emphasis of CBR and DID is 'upstream' agendas of management, policy and international declarations. Such priorities primarily reflect the interests and priorities of some NGOs and international bureaucrats rather than people with disabilities in the global South. We also contend that the evolving nature of CBR has made it quite ill-defined, resulting in a lack of identity and direction in some crucial dimensions. We suggest that adverse aspects of this unplanned evolution which characterizes CBR include limited organizational leadership, inadequate global recognition, the predominance of western values, poor educational and research infrastructure, and a meagre funding stream. These issues have stunted the development of a theoretical framework or robust academic analysis. Practically, this has also hindered the clear elucidation of a leadership structure and training framework for CBR. The current reality is that the global CBR movement has not grown commensurate with the number of people in the global South who require the services, empowerment, livelihoods and social inclusion that it should facilitate.

\section{The Evolving Identity of CBR}

Given the focus on local resources, family member skills and the distinctive characteristics of local communities, it is not surprising that the implementation of CBR was never uniform and was commonly adapted, resulting in a diverse and evolving model of practice (Kuipers 1998). On the positive side, this has resulted in an approach which is readily adaptable, with 
great potential to be contextually responsive (Kuipers 1998). In some settings the model has evolved constructively in response to the local context (Werner 1993) and is increasingly being promoted by NGOs and by governments in the global South (CCBRT 2014). Such evolution has created the potential for greater relevance, attentiveness to local culture, and responsiveness to socio-economic realities.

However, this evolution has been inconsistent across settings and across dimensions of CBR. Some examples of CBR practice have evolved very little from their western origins in the 1970s, and maintain stereotypical treatment programmes, regardless of need or context (Giacaman 2001). Other aspects have evolved considerably, but with questionable outcomes for the majority of people with disabilities. The mercurial nature of CBR, without clear models, has permitted potentially useless or even unhelpful structures and practices to flourish in some settings. For example, in a review of 37 CBR evaluation reports, weaknesses in management and unhelpful management practices were seen as the primary cause for concern by evaluators (Kuipers et al. 2008). Such practices presumably emerged in the absence of clear management guidelines, as CBR evolved from a set of rehabilitation techniques, rather than from a considered organizational and management foundation. While key international CBR agencies have now produced formal management guidelines, and a framework for CBR is now in place (WHO et al. 2010), this wasn't undertaken until 30 years after the initial promotion of the CBR model.

The indistinct and evolving nature of CBR is not just a historical phenomenon, but is also a defining characteristic of the model. This is well illustrated in the widely used CBR Matrix (Figure 1), which accompanied the CBR Guidelines (WHO et al. 2010). The matrix suggests that the scope of CBR is now so broad and all-encompassing that it is almost indefinable. The CBR Matrix consists of five key components (columns) each comprising five elements (rows). Four components (Health, Education, Livelihood, and Social) relate to development 
sectors; the fifth, Empowerment, addresses access to development. The elements under each key component describe the range of options that could be implemented as CBR. Such breadth, coupled with the evolving nature of CBR, has made it very difficult to define, to investigate and particularly to evaluate the impact and outcomes of the model. As a result, questions of quality of services and cost-effectiveness of CBR have essentially not been asked, evidence of outcomes has not been demonstrated, and rigorous comparative analysis at an international level has not yet occurred (Hartley et al. 2009; Thomas and Thomas 1999).

\section{[insert Figure 1 here]}

\section{The Voice of People with Disabilities in the Identity of CBR}

From relatively early, a consistent critique of CBR internationally has been that people with disabilities have had very limited voice in the conceptualization of CBR services and models (Werner 1995), or in the management and running of CBR programmes (Lang 1999). In many historical and contemporary CBR projects, power resides with the professionals who manage the service, and the role of people with disabilities is as passive 'recipients' or 'beneficiaries' (Lang 2000). While these critiques remain pertinent in many CBR settings globally, and the voice and leadership of people with disabilities remains very limited, there are signs of constructive directions emerging. New training approaches have the potential to challenge traditional professional practices (Rule 2013), and new models of service delivery are evolving, which will lead to a reconceptualization of roles (Lang 2011). Such emerging models have the potential to shape CBR and DID in encouraging new ways. 


\section{The Identity of CBR Among DPOs}

The decades in which CBR emerged have also witnessed the rise of disabled people's organizations (DPOs) in western nations as well as in the global South. In many instances, relationships between CBR service providers and DPOs have been difficult. In some instances, CBR implementing organizations have seen DPOs as peripheral and either failed to engage or actively ignored them. Conversely some DPOs in the global South, which have tended to be city-based, have viewed CBR as an outdated service delivery system, suited only to rural areas (Thomas 2011).

At the implementing country level, early relationships between internationally funded and professionally supported (and usually foreign) CBR organizations and local, unfunded DPOs were quite uneasy (S. Miles 1996). In part this was due to the attention given to the CBR organizations by local government officials as a result of the funding that accompanied their work. More recently this situation is starting to change. With interest among international donors and aid agencies increasingly shifting towards directly funding and supporting DPOs, the identity of CBR and the relationship between CBR organizations and DPOs is further being re-examined.

Some CBR leaders have advocated that the roles of CBR organizations and DPOs should increasingly be integrated at all levels (WHO 2003). Indeed, some advocate that CBR projects should instigate and strengthen DPOs, and that DPOs can also initiate and run CBR programmes (Deepak et al. 2013). This shift, in combination with the interest from donors to fund DPOs, means that they may increasingly become service providers, and that CBR organizations may see some of their area of influence diminish.

The diminution of influence of some CBR NGOs, and the potential for DPOs to become providers of rehabilitation or disability services may be a preferred model for many. Under such a structure people with disabilities may have a greater voice in management, service 
provision and in the emergence of more appropriate services. This emerging direction, however, also holds the potential for both CBR organizations and DPOs to lose their respective distinctive features and identities. For example, if DPOs become service providers, they may lose some of the 'moral authority' they hold as independent advocates and watchdogs. IThey may easily become conflicted, and may no longer have the necessary independence to hold service providers accountable. A potential direction for more constructive evolution may be for CBR programmes to build the capacity of people with disabilities and their families through self-help groups, which are then linked with independent DPOs, advocating to governments and service providers and providing different forms of support and capacity building (Suharto et al. 2013).

\section{The Identity of CBR and Fundamental Values}

As implied earlier, the evolving and adaptable nature of CBR has not necessarily been manifest in all dimensions of the approach. For example, a consistent critique of the early CBR model was that it naively conformed to traditional, medicalized approaches to rehabilitation (Gatjens 2009). While this is less an issue in contemporary CBR practice, there are a number of other aspects of western conceptualization which have also been adopted without much adaptation to the diverse contexts of the global South. For example, it has been observed in the South Asian context that proponents of CBR and DID inappropriately impose a narrow western perspective of disability development as a human rights issue, regardless of context or the strengths and weaknesses of communities (M. Miles 1996). This would appear to be an example of a western colonial mindset, but in a less overt form, since such issues are seen by many in the CBR world as beyond debate or discussion.

Likewise, proponents of CBR have strongly emphasized conventional notions of empowerment of people with disabilities: from early publications (Helander 1999) to more 
recent frameworks (WHO et al. 2010). Western concepts of individual 'empowerment', however, are often perceived as selfish in some Asian contexts, and inconsistent with the philosophy of ubuntu (human interconnectedness) in some African contexts. In many developing country settings, the concerns of the family or community have precedence over individual empowerment, and indeed in some societies such notions of empowerment may be perceived as a threat to positive social order and community harmony (Thomas 2011). While such differences are not new or even necessarily contradictory, the CBR movement has not had a robust debate as to the relevance, adaptation or reinvention of concepts such as human rights, empowerment or even community-based action (Kuipers 2014). In particular, for many women with disabilities in the global South, these western notions are peripheral at best, or at times major obstacles to achieving their aspirations for greater collective identity and growth (Lorenzo 2003).

The tendency within CBR and DID of directly transposing western notions of empowerment, rights or other values may be seen as akin to the practice of transposing western models of rehabilitation in the 1970s. As in the '70s, some contemporary western disability activists and academics appear resolute that post-enlightenment and post-modern conceptualizations should be adopted post-haste, regardless of context. Indeed, it is only in recent years that distinctly global South voices and models are being proposed for disabilityrelated action and research (Mji et al. 2011). Without such critical analysis and debate arising from the global South, in directly importing such concepts without due regard to local contexts, cultures, beliefs and values, CBR is unlikely to advance in a contextually sustainable way. 


\section{The Identity of CBR and Religion}

The roll out of the CBR approach also appears to have strongly adhered to western secular conceptualizations and sensitivities, deliberately ignoring issues of religion and spirituality (Crishna 1999; see also Betcher and Wangila 2016, in this volume). While religion has occasionally been acknowledged as a contextual issue in the Guidelines (WHO et al. 2010), the importance or potential of religion is not addressed in official CBR documents in any meaningful way. Recognizing that religion is a critical source of meaning and identity for the majority of people in the global South, and that disability is immersed in this meaning system (Grech 2012), this would seem a curious omission.

In part, this omission stems from the medical conceptualization that was so fundamental in the evolution of CBR. Western science-based, medical and rehabilitation models have developed away from, and have often been in conflict with, traditional religious belief systems (M. Miles 2010). This has aligned with western fixations on secular and individualistic conceptualizations in development circles (Grech 2012). As a result, the CBR world has not had serious discussion about the incorporation of faith, spirituality, beliefs and religion into practice. Issues such as karma, destiny, fate, or the Islamic concept of taqdeer, have rarely, if ever, been discussed. Moreover, potential connections between religious beliefs and wellbeing, hope, quality of life, resilience, or even the supportive and affirming potential of communities of faith and religious congregations (Grech 2012), have largely been ignored in the CBR discourse.

Recognizing the fundamental importance of religion and belief in the lives and communities of most people in the global South (Grech 2012), and that many western CBR NGOs (such as CBM, World Vision, Caritas, and numerous leprosy organizations) have come from faith-based frameworks, the silence on these issues is surprising. While they may be somewhat contentious from a western secular perspective, without robust discussion and 
ongoing acknowledgement of these issues, the field of CBR is unlikely to evolve in ways that touch the lives and are coherent with the values, of the majority of people and communities in the global South.

As the emphasis in CBR evolves towards a more inclusive development approach, the prospects for addressing this shortcoming are not encouraging. The DID approach with a single-minded emphasis on rights, conventions, policy and legislation would equally appear to have little to contribute to these issues. As Grech has noted, rights, policies and legislation have replaced God in the secular conceptualization. In combination with empowerment and socio-economic advancement, they are the means by which redemption is gained (Grech 2012) in the new frameworks being imposed on people with disabilities in the global South through the DID model.

\section{The Identity of CBR and Gender}

Encouragingly, contemporary CBR literature consistently mentions the issue of gender. The need to consider gender issues and the importance of gender equality are noted throughout the new Guidelines (WHO et al. 2010) and other more recent publications (Ghosh 2011). In most instances these statements are instructions for CBR managers to support the full participation of women and girls. Given the complexity and importance of gender issues in the global South, however, this level of analysis would seem somewhat superficial. We suggest that in the emerging identity of CBR, gender issues warrant much more detailed attention and nuanced analysis.

First, it is important to distinguish between sex (bio-physiological characteristics which differentiate men and women) and gender (socially constructed behaviours, expectations and roles that derive from, but may not depend on sex) (Vlassoff and Manderson 1998). Given the socially and culturally defined nature of gender, notions of gender roles, preferred roles or 
even of gender equality cannot simply be transferred from one culture to another (Mohanty 1988). Unfortunately, perspectives of roles, gender and equality in global South cultures and communities are often widely discrepant from those of western CBR professionals and NGOs (Sabuni 2004). This issue has proven somewhat problematic in CBR, which in advocating gender equality may be seen as reflecting western views of gender. In so doing CBR may actually perpetuate western hegemonic structures and exacerbate inequalities (Mohanty 1988) by imposing western assumptions on families and communities of the global South.

Next, the CBR model includes numerous inherent assumptions which profoundly affect the role and place of women in settings where it is introduced. For example, most forms of the CBR model reflect an unwritten expectation of the role of women as carers (often of children with a disability). This may be seen to perpetuate the role of women in the unpaid private or domestic sphere and contribute to their marginalization and social exclusion. By relegating women to such prescribed roles they are excluded from the labour force and other important social areas (Giacaman 2001) and the role of men is reinforced in the paid public sphere (England et al. 2002). Such reinforcement of gender roles substantially diminishes the potential role women might play in economic and other forms of development (Boserup et al. 2013).

Similarly, the CBR model relies heavily on community meetings, community-level committees, and the dissemination of information (WHO et al. 2010). In many societies in the global South, women do not participate in such meetings or formal committee structures and decision making (Sabuni 2004), and are more likely to be illiterate (Stromquist 1990). As such this may be seen as another way the CBR model perpetuates the marginalization of women, removing them from decision making for an activity in which their work is actually vital. 
Paradoxically, the influence of CBR on gender issues also has numerous positives. CBR engagement may create opportunities for women and girls, promoting their involvement in development activities from which they would otherwise be excluded. Indeed there is evidence that participation in CBR initiatives actually achieves substantial outcomes for women, playing a key role in their social, emotional and economic development (Lorenzo 2003).

We argue that the complexity of engaging with patriarchal social structures, the valuing and remuneration of tasks, and particularly the valuing of disability and rehabilitation support activities, requires substantial consideration and exploration that has yet to occur in CBR. Likewise, for people with disabilities, gender issues are contentious and deserve more serious attention in the emerging models of CBR. For example, ensuring that people with disabilities attain livelihood support in gender-valued roles may not be a primary concern for CBR providers, but may be very important to all other stakeholders, particularly the person with a disability.

As a final example, in post-conflict CBR settings, the relative numbers of men and women with disabilities, and the nature of their disabilities, change in dramatic and complex ways. In many such societies the esteem given to war veterans, and their capacity for advocacy, may be very different from those with other disabilities. The field of CBR has yet to deal with these issues conclusively or to plan models of CBR accordingly. For CBR to evolve further on gender issues, more innovative and contextually responsive analysis is required.

\section{The Identity of CBR Research and Education}

Another challenge to the identity of CBR has been that no substantial academic and research leadership has evolved within the movement. A key characteristic of CBR is the surprising lack of dedicated research centres or academic departments focused on this area. Irrespective 
of the kind of research, methodology or orientation, the most commonly noted critique of CBR is that it lacks a substantial research foundation (Finkenflugel et al. 2005; Velema et al. 2008). Similarly, academic commitment to the education and training of CBR practitioners, and the development of CBR managers has been very limited.

For a number of years the Centre for International Child Health (as it was then called) at University College London and the International Centre for the Advancement of CBR at Queens University, Kingston, Ontario were among the only centres with a recognizable research and academic focus on CBR, but both have struggled to remain viable, to gain recognition and to achieve minimum required funding. Beyond those few centres, the dispersed academics and research-interested practitioners from elsewhere in the world have not been able to reach any substantial critical mass or momentum, nor foster a sustainable research culture to advance CBR.

The reasons for this limitation may stem from the evolution of CBR based on a manual of practical strategies to be used by family members and volunteers in the global South. This 'grab bag' of simple techniques, lacking a philosophy or coherent theory, has held little interest for conceptually oriented research institutions. Other universities and more applied research centres have similarly shown limited interest, presumably due to the absence of potential funding streams. Despite vast need and enormous potential, the world of CBR research does not have powerful patrons such as drug companies, and appears not to have fallen within the purview or key priorities of United Nations (UN) agencies or the World Bank.

Further, with no prospect of educating large numbers of fee-paying or western students in degree programmes, the potential interest of training universities has been minimal. While the educational needs within CBR are equally enormous (Mannan et al. 2013), they exist in the 
global South, are often at a basic level, and typically comprise hands-on learning, short courses or on-the-job skill development.

As noted, within development circles (and including university departments of international development), aid agencies and major funding bodies such as the World Bank have shown limited interest in CBR. It would appear that CBR is seen as having limited relevance to their organizational objectives and lacking alignment with structural adjustment priorities or their economic priorities. This in turn has limited the potential for funding of commissioned research and evaluations on which such departments depend. Likewise, the potential support for research from CBR implementing agencies has been limited. Individual donors to such agencies are typically motivated to practically assist people with disability in the global South. In such cases, the support of academic aspirations, no matter how functional, may be seen as a luxury against the needs of individuals being emphasized by the agency.

In any field of life and endeavour, and particularly one which addresses such important issues as disability and poverty, meaningful research, critique, evidence, analysis and innovation are vital to healthy growth. For the majority of the history of CBR, this has not been the case. Serious critique and academic reflection pertaining to disability and development have mostly existed beyond the field of CBR (Priestley 2001; MacLachlan and Swartz 2009). While some academic centres and alliances have recently emerged (and encouragingly, some are emerging in the global South), they likewise continue to struggle; as reflected in the recent loss of the CBR specialty at Makerere University, Uganda. The identity of CBR depends to a considerable extent on the establishment of broad and diverse centres of research and education excellence, particularly in the global South. 


\section{The Identity of CBR in the Context of International Agencies}

While the evolving and amorphous nature of CBR has been one factor constraining its advancement and improvement over the decades, another has been the neglect of disability and CBR concerns by the most important international agencies. Historically, UN agencies, the World Bank and similar bodies appear to have been indifferent to disability and CBR issues. For economically focused bodies and monetary funds, CBR as a collection of techniques for marginalized people, most of whom are not economically active, would appear to have been immaterial. For health-related agencies such as the WHO, CBR does not result in vaccines or surgical cures, and does not operate in the realm of life-saving technology or medications. Comprising relatively simple activities with people and families who are unlikely to see dramatic medical recovery, CBR has clearly not cornered the organizational priorities of these key players. For example, comparing the UN agency infrastructure for CBR with the UN service response to people with HIV/AIDS is striking. In Geneva, the Disability and Rehabilitation team tasked with this work comprises a handful of people who occupy a small section of a back corner of one floor of the WHO building. Their role is to facilitate a key dimension of the UN's response to the 190 million people who have significant disabilities globally. Within that handful of people, only a couple of individuals have some responsibility for promoting CBR as the primary global service delivery strategy. The contrast with the UN response to the HIV/AIDS crisis is stark. This condition, which affects far fewer people globally, warrants its own UN department (in addition to an array of offices in other UN agencies). It occupies a multistorey building with many hundreds of employees in Geneva alone. The UN service response is a multi-agency response with a multibillion dollar budget. Obviously the disastrous HIV/AIDS global epidemic is devastating to millions of lives and communities, and warrants urgent and concerted attention. However, the irony of comparing the potential UN leadership of that service 
response with the capacity of the WHO Disability and Rehabilitation team to advocate and support CBR services indicates the challenge faced in forming the identity and underlining the relevance of CBR. The failure of CBR to engage with major systemic and structural agendas at the international level may in part be attributed to an approach which has evolved passively over the decades, without clear leadership and intention.

\section{The 'NGO’ Identity of CBR}

Related to the weak identity of CBR at the international level is the slow uptake of CBR by governments in the global South. Despite nearly 40 years of activity, CBR projects are still largely implemented and sustained by international disability NGOs. The underlying NGO commitment to transitioning CBR projects to government departments of health, education, social welfare or labour have only been realized in a few cases. Instances of governments adopting and sustaining the CBR infrastructure developed by NGOs are very limited.

The causes of this enduring dependence on NGOs are numerous. They may stem from a reluctance for NGOs to fund local agencies or grassroots initiatives; they may also stem from the creation of a culture of dependence, or possibly from the imposition of a foreign model that is simply not sustainable without significant and ongoing NGO assistance. Alternatively it may stem from local governments not seeing people with disabilities as a priority. Regardless of the cause, a consequence of being predominantly implemented by NGOs is that CBR has nearly always been implemented in the form of specific 'projects'. CBR initiatives are typically micro-level activities in discrete areas such as a rural area, a slum, or a district. Further, such projects are almost always time-limited. The identity and reality of CBR in the form of micro-level, time-limited projects runs contrary to the needs of people with disabilities in countries of the global South. For CBR to have an impact on the lives of people 
with disabilities and their families, there is a need for service initiatives to be ongoing and at a national scale, and with maximum coverage.

While CBR remains predominantly the domain of external agencies, it will be difficult for people with disabilities (and for implementing organizations) to tap into the political will, or to influence major government agendas in the global South. Even though such CBR projects may succeed at an individual level, restoring function and building participation, they will continue to only partially address many of the detrimental contextual factors that influence disadvantage (Thomas and Thomas 1999).

\section{The Changing Identity of CBR: Evolving Towards DID}

Now almost four decades since the beginning of the WHO initiative, the CBR paradigm is undergoing further change of identity in the form of a major shift of perspective and terminology. In many CBR circles, the terms 'inclusive development' or 'disability-inclusive development’ are replacing CBR.

For some advocates, DID is a radically new paradigm, with a focus on making mainstream development inclusive of all people with disabilities. In this reconceptualization, the focus is not the provision of rehabilitation, or the individual person with a disability, or even their family. In such cases, DID is the application of principles of participatory community development, to maximize the inclusion, self-determination, participation of, and social justice for, people with disabilities. In other examples, the new models of DID appear more like the simple renaming of old CBR practices. Between the two poles, some CBR proponents emphasize that CBR is a strategy and that DID is the goal (Khasnabis 2011). In this more nuanced approach, inclusive development is the desired end result (making communities and society at large inclusive of all marginalized groups, including people with 
disability) (Thomas 2011), and CBR is a key means of attaining that end result. Such a framework may finally provide CBR with the clarity of identity and form it needs.

The shift in emphasis towards DID may be a very constructive step towards addressing some of the weaknesses of CBR, such as its 'micro' focus and failure to tackle contextual and systemic issues that affect people with disability in the global South. However, many CBR proponents and other stakeholders (Corneilje and Veldman 2011) would argue that this activity must complement the practical, physical and micro focus of people with disabilities and their families.

If CBR is to play a key part in a constructive or preferable model of disability services for people with disability in the global South, it should work more clearly with people with disabilities to address their expressed needs, build their capacity, and support their families. Within the development sector, it should ensure equal opportunities and rights, and facilitate people with disability and their families to become self-advocates for inclusion in all development processes. At the social level, it should work with the community and society at large to remove barriers that exclude people with disability. Whether such a model can evolve out of the CBR context is an issue on which the lives and aspirations of millions of people with disability in the global South depend.

\section{Conclusion}

The importance of CBR in the global South is clear. The World Report on Disability (WHO and World Bank 2011) acknowledges CBR "as one of the significant responses to address concerns related to access to services, opportunities, participation and inclusion of persons with disabilities" globally. A key question is whether recent developments in the evolution of CBR and DID, which include the CBR Guidelines, the CBR Matrix, and the adoption of 
disability-inclusive development as a new paradigm, will provide enough vision and direction to address the numerous limitations outlined in this chapter.

We suggest that the primary risk with these new directions and documents is that the current situation for people with disabilities in the global South will not change dramatically. First, all of the major responses and documents that have emerged in recent years have been clearly focused on 'upstream' agendas. They seek to address the limitations on CBR by looking at big picture conceptual issues, management concerns and human rights issues. Clearly these are important, but they also have limitations. For example, the breadth of the CBR Matrix may not assist in sufficiently defining and orienting CBR around the concerns of people with disabilities in the global South; the CBR Guidelines may not address the practical needs of people with disabilities; and the shift towards disability-inclusive development may result in services to people with disabilities in the global South becoming increasingly diluted across numerous international aid and development agendas. The extent to which the CBR and DID models of the future can also focus 'downstream' is a key issue. The extent to which these evolving directions can meet the practical needs of people with disabilities, foster their social participation and inclusion, address gender concerns, incorporate a human rights framework, and ensure that people with disabilities are central and active partners in decisions that impact on their lives, remains to be seen.

\section{References}

Betcher, S., \& Wangila, M.N. (2016) Religion after the medical miracle: recovering 'disability' as religious analytic of social suffering. In S. Grech \& K. Soldatic (Eds), Disability in the Global South: The Critical Handbook (pp. xx-Xx). New York: Springer.

Boserup, E., Tan, S. F., Toulmin, C. (2013). Woman's role in economic development. London: Routledge.

CBM. (2014). Disability \& development work. http://www.cbm.org/Disability-and-development-work250259.php. Accessed 3 May 2014.

CCBRT. (2014). Comprehensive community based rehabilitation in Tanzania. http://www.ccbrt.or.tz/home/. Accessed 3 May 2014.

Corneilje, H., \& Veldman, E. (2011). The dream of inclusion for all: Powerful CBR training materials. Alphen ann den Rijn, NL: Enablement. 
Crishna, B. (1999). What is community-based rehabilitation? A view from experience. Child: care, health and development, 25(1), 27-35. doi: 10.1046/j.1365-2214.1999.00087.x.

Deepak, S., dos Santos, L. R., Griffo, G., de Santana, D. B., Kumar, J., Bapu, S. (2013). Organisations of Persons with Disabilities and Community-based Rehabilitation. Disability, CBR \& Inclusive Development, 24(3), 5-20.

England, P., Budig, M., Folbre, N. (2002). Wages of virtue: the relative pay of care work. Social Problems, 49(4), 455-473. doi: 10.1525/sp.2002.49.4.455.

Evans, P. J., Zinkin, P., Harpham, T., Chaudury, G. (2001). Evaluation of community-based rehabilitation for disabled persons in developing countries. Social Science \& Medicine, 53(3), 333-348. doi: 10.1016/S02779536(00)00321-X.

Finkenflugel, H., Wolffers, I., Huijsman, R. (2005). The evidence base for community-based rehabilitation: a literature review. International Journal of Rehabilitation Research, 28(3), 187-201.

Gatjens, L. F. A. (2009). A basic manual for inclusive development. Nicaragua: Handicap International and Inter-American Institute on Disability and Inclusive Development. http://www.handicapinternational.fr/fileadmin/documents/publications/BasicManualID.pdf. Accessed 11 August 2014.

Ghosh, N. (2011). Challenging gender ideologies through CBR. http://www.cbrglobal.org/Downloads/CS5.2.1.pdf. Accessed 11 August 2014.

Giacaman, R. (2001). A community of citizens: disability rehabilitation in the Palestinian transition to statehood. Disability \& Rehabilitation, 23(14), 639-644. doi: 10.1080/09638280110036544.

Grech, S. (2012). Disability and the majority world: a neocolonial approach. In D. Goodley, B. Hughes \& L. Davis (Eds), Disability and social theory: new developments and directions (pp. 52-69). Basingstoke: Palgrave Macmillan.

Handicap International. (2014). Community based rehabilitation. http://www.handicapinternational.org.uk/what_we_do/rehabilitation/community_based_rehab. Accessed 3 May 2014.

Hartley, S., Finkenflugel, H., Kuipers, P., Thomas, M. (2009). Community-based rehabilitation: opportunity and challenge. Lancet, 374(9704), 1803-1804. doi: 10.1016/S0140-6736(09)62036-5.

Helander, E. (1999). Prejudice and dignity: An introduction to community-based rehabilitation. Geneva: World Health Organization.

Helander, E., Mendis, P., Nelson, G., Goerdt, A. (1989). Training in the community of people with disabilities. Geneva: World Health Organization.

IDDC. (2010). Contribution: OHCHR, Human Rights Council Resolution 13/11, 'Human rights of persons with disabilities'. Brussels: International Disability and Development Consortium. www2.ohchr.org/english/issues/disability/docs/study/IDDC.doc. Accessed 3 May 2014.

ILO, UNESCO, \& WHO. (2004). CBR: a strategy for rehabilitation, equalization of opportunities, poverty reduction and social inclusion of people with disabilities. Joint position paper. Geneva: ILO, UNESCO and WHO.

Khasnabis, C. (2011). Community-based rehabilitation (CBR) - a common strategy in the developing world for rehabilitation, poverty reduction and promotion of human development. Presented at Inclusive early childhood development - an underestimated component within poverty reduction, Bonn, Germany, 3-4 February.

Kuipers, P. (1998). Community based rehabilitation (CBR) as engagement: context, parameters and potential. PhD thesis. Brisbane: Griffith University.

Kuipers, P. (2014). Empowerment in community-based rehabilitation and disability-inclusive development. Disability, CBR \& Inclusive Development, 24(4). http://dcidj.org/article/view/274.

Kuipers, P., Wirz, S., Hartley, S. (2008). Systematic synthesis of community-based rehabilitation (CBR) project evaluation reports for evidence-based policy: a proof-of-concept study. BMC International Health and Human Rights, 8(3). doi: 10.1186/1472-698X-8-3.

Lang, R. (1999). Empowerment and CBR? Issues raised by the South Indian experience. In E. Stone (Ed.), Disability and development: Learning from action and research on disability in the majority world (pp. 130148). Leeds: The Disability Press.

Lang, R. (2000). The role of NGOs in the process of empowerment and social transformation of people with disabilities. In M. Thomas \& M.J. Thomas (Eds.), Selected readings in community based rehabilitation (pp. 1-20). Bangalore: Occasional Publication of the Asia Pacific Disability Rehabilitation Journal.

Lang, R. (2011). Community-based rehabilitation and health professional practice: developmental opportunities and challenges in the global North and South. Disability \& Rehabilitation, 33(2), 165-173. doi:10.3109/09638288.2010.487923.

Lorenzo, T. (2003). No African renaissance without disabled women: a communal approach to human development in Cape Town South Africa. Disability \& Society, 18(6), 759-778. doi: 10.1080/0968759032000119505. 
MacLachlan, M., \& Swartz, L. (2009). Disability \& international development: Towards inclusive global health. New York: Springer.

Mannan, H., MacLachlan, M., McAuliffe, E. (2013). The human resources challenge to community based rehabilitation: the need for a scientific, systematic and coordinated global response. Disability, CBR \& Inclusive Development, 23(4), 6-16. http://dcidj.org/article/view/157.

Miles, M. (1996). Community, individual or information development? Dilemmas of concept and culture in South Asian disability planning. Disability \& Society, 11(4), 485-500. doi: 10.1080/09687599627552.

Miles, M. (2010). Religion and spirituality. In J. Stone \& M. Blouin (Eds.), International encyclopedia of rehabilitation. Buffalo: CIRRIE. http://cirrie.buffalo.edu/encyclopedia/en/article/1/. Accessed 11 August 2014.

Miles, S. (1996). Engaging with the Disability Rights Movement: the experience of community-based rehabilitation in southern Africa. Disability \& society, 11(4), 501-518. doi: 10.1080/09687599627561.

Mji, G., Gcaza, S., Swartz, L., MacLachlan, M., Hutton, B. (2011). An African way of networking around disability. Disability \& Society, 26(3), 365-368. doi: 10.1080/09687599.2011.560419.

Mohanty, C. T. (1988). Under western eyes: feminist scholarship and colonial discourses. Feminist Review, 30, 61-88. http://www.jstor.org/stable/1395054.

Priestley, M. (2001). Disability and the life course: Global perspectives. Cambridge: Cambridge University Press.

Rule, S. (2013). Training CBR personnel in south africa to contribute to the empowerment of persons with disabilities. Disability, CBR \& Inclusive Development, 24(2), 6-21. http://dcidj.org/article/view/180.

Sabuni, L. P. (2004). Gender differences in attitudes and practices within households in rural areas of the north east of the Democratic Republic of Congo. Liverpool: University of Liverpool.

Stromquist, N. P. (1990). Women and illiteracy: the interplay of gender subordination and poverty. Comparative Education Review, 34(1), 95-111. http://www.jstor.org/stable/1188557.

Suharto, S., Dorsett, P., Kuipers, P. (2013). Encouraging 'difability' thinking in community based rehabilitation: addressing poverty and social exclusion. Presented at Development futures: alternative pathways to end poverty, Sydney, 21-22 November. http://www.slideshare.net/DevFutures/suharto-suharto-communityexclusion-29437895\#. Accessed 11 August 2014.

Thomas, M. (2011). Reflections on community-based rehabilitation. Psychology \& Developing Societies, 23(2), 277-291. doi: 10.1177/097133361102300206.

Thomas, M., \& Thomas, M. J. (1999). A discussion on the shifts and changes in community based rehabilitation in the last decade. Neurorehabilitation and Neural Repair, 13(3), 185-189. doi: 10.1177/154596839901300308.

Velema, J. P., Ebenso, B., Fuzikawa, P. L. (2008). Evidence for the effectiveness of rehabilitation-in-thecommunity programmes. Leprosy Review, 79(1), 65-82.

Vlassoff, C., \& Manderson, L. (1998). Incorporating gender in the anthropology of infectious diseases. Tropical medicine \& international health: TM \& IH, 3(12), 1011-1019.

Werner, D. (1993). Meeting the needs of disabled village children. Tropical and geographical medicine, 45(5), 229-232.

Werner, D. (1995). Strengthening the role of disabled people in community based rehabilitation programmes. In B. O’Toole \& R. McConkey (Eds), Innovations in developing countries for people with disabilities. Chorley: Lisieux Hall.

Werner, D. (1999). Disabled village children. Palo Alto, CA: Hesperian Foundation.

WHO. (2003). International consultation to review community-based rehabilitation (CBR). http://whqlibdoc.who.int/hq/2003/who_dar_03.2.pdf. Accessed 11 August 2014.

WHO, \& World Bank. (2011). World report on disability. Geneva: World Health Organization. http://www.who.int/disabilities/world_report/2011/report/en/. Accessed 11 August 2014.

WHO, UNESCO, ILO, IDDC. (2010). CBR guidelines. Geneva: World Health Organization.

\section{Figure captions}

Figure 1. CBR Matrix 